\title{
Epitope topology and removal of mouse acrosomal plasma membrane by P12-targeted immunoaggregation is
}

\author{
Han-Jia Lin ${ }^{\text {a }}$, Ching-Wei Luo ${ }^{\mathrm{b}}$, Chia-Yih Wang ${ }^{\mathrm{c}}$, Yee-Hsiung Chen ${ }^{\mathrm{c}, \mathrm{d}, *}$ \\ ${ }^{a}$ Institute of Bioscience and Biotechnology, National Taiwan Ocean University, Keelung, Taiwan \\ ${ }^{\mathrm{b}}$ Faculty of Life Sciences and Institute of Genome Sciences, National Yang-Ming University, Taipei, Taiwan \\ ${ }^{\mathrm{c}}$ Institute of Biochemical Sciences, College of Science, National Taiwan University, Taipei, Taiwan \\ ${ }^{\mathrm{d}}$ Institute of Biological Chemistry, Academia Sinica, Taipei, Taiwan
}

Received 4 August 2006

Available online 18 August 2006

\begin{abstract}
P12 is a Kazal-type trypsin inhibitor that has been purified from mouse seminal vesicle secretion. We observed a slight impact of P12 on sperm capacitation, and demonstrated the removal of plasma membrane overlaying the acrosome region by immunoaggregation of P12 on mouse sperm. Further, we compared the immunoreactivity of P12 antibody to ten P12 variants, including six single-site mutated mutants (R19L, Y21V, D22G, R43G, K44S, and R45T), two multisite mutated mutants (R43G/K44S/R45T and L50H/R52G/K53A), and two deletion mutants ( $\mathrm{Nd} 10$ and $\mathrm{Cd} 8)$ in which 10 and 8 residues were deleted from the $\mathrm{N}$ - and C-terminals, respectively. We found that the $\mathrm{N}$-terminal region, ${ }^{43} \mathrm{RKR}^{45}$, and the $\mathrm{C}$-terminal region, but not $\mathrm{R}^{19}, \mathrm{Y}^{21}$, and $\mathrm{D}^{22}$, are involved in the three epitopes that reside on one side and are three-dimensionally distant from $\mathrm{R}^{19}, \mathrm{Y}^{21}$, and $\mathrm{D}^{22}$ on the P12 molecule. Based on the epitope topology, we elucidated the structural basis by which P12 antibody immunoaggregated P12 on sperm head.
\end{abstract}

(C) 2006 Elsevier Inc. All rights reserved.

Keywords: Acrosomal removal; Epitope; Immunoagglutination; Protease inhibitor

Studying protease inhibitors in the genital tract of mammals is an important subject in molecular reproduction because they are believed to be important for the protection of genital tract epithelium against the damage by proteolysis [1] and for the regulation of the fertilization process $[2,3]$. Seminal vesicle, a male mammalian accessory apparatus, is observed in many mammalian species. After puberty, seminal vesicle secretion (SVS) accumulates in the lumen of this sexual gland. Upon ejaculation, SVS is squirted into urethra to make up the major part of seminal plasma, which is the complex biological fluid formed from the mixing of various fluids and is discharged from the male reproductive tract. It has been reported that the fertility of mice

\footnotetext{
This work was supported in part by Grants 95-3112-B-002-024 and 942311-B-002-004 from the National Science Council, Taipei, Taiwan.

* Corresponding author. Fax: +886223635038.

E-mail address: bc304@gate.sinica.edu.tw (Y.-H. Chen).
}

as well as rats is greatly reduced if their seminal vesicles are extirpated or removed [4,5], manifesting the importance of this accessory sexual gland in reproduction. Previously, we purified a Kazal-type trypsin inhibitor consisting of 57 amino acid residues from mouse seminal vesicle secretion (SVS) [6]. This rather small protein was tentatively named as P12 because it is derived from P12 cDNA that was cloned from the mouse ventral prostate by Mill et al. [7]. Among the mouse sexual glands, P12 is exclusively expressed in seminal vesicle, coagulating gland and prostate of adult males. Because P12 appears only in trace amount in mouse SVS, the need for a significant quantity of P12 for the study of its structure-function relationship prompted us to produce a recombinant P12 with the full activity of the naturally occurring P12 from a chimeric polypeptide of glutathione S-transferase and P12 (GSTP12) expressed in Escherichia coli [8]. Our study on P12sperm binding demonstrated a single-type P12-binding site 
$\left(1.49 \times 10^{6}\right.$ site/cell $)$ with a $K_{\mathrm{d}}$ value of $70 \mathrm{nM}$ on the membrane overlaying the anterior acrosome of mouse sperm; we also showed an inhibitory effect of $\mathrm{P} 12$ on $\mathrm{Ca}^{2+}$ uptake by mouse sperm [9]. Further, we distinguished $\mathrm{R}^{19}$ on $\mathrm{P} 12$ as the reactive site for trypsin inhibition compared to $\mathrm{D}^{22}$ and/or $\mathrm{Y}^{21}$, which are mainly responsible for the binding of P12 to sperm [10]. We conducted this work to understand the topology of epitopes, $\mathrm{R}^{19}, \mathrm{D}^{22}$, and $\mathrm{Y}^{21}$ on the $\mathrm{P} 12$ molecule in order to elucidate the structural basis of how the P12-sperm binding leads to the removal of plasma membrane overlaying acrosome region by immunoaggregation of $\mathrm{P} 12$ on the mouse sperm.

\section{Materials and methods}

Preparation of P12 and the recombinant P12 variants. Outbred CD-1 mice were purchased from Charles River Laboratories (Wilmington, MA) and were maintained and bred in the animal center at the College of Medicine, National Taiwan University. Animals were treated in a manner that followed the institutional guidelines for the care and use of experimental animals. They were housed under controlled lighting (14 h light, $10 \mathrm{~h}$ dark) at $21-22^{\circ} \mathrm{C}$ and were provided with water and NIH 31 laboratory mouse chow ad libitum. Adult mice (8-12-weeks-old) were sacrificed by cervical dislocation. Seminal vesicles were carefully dissected to free them from the adjacent coagulating glands, and the secretion collected from 50 mice was placed directly into $50 \mathrm{ml}$ of ice-cold $5 \%$ acetic acid. P12 was purified from the SVS according to a previously described procedure $[6,11]$.

Site-directed mutagenesis for P12 generally followed the instructions of Promega Altered Site II in vitro Mutagenesis System kit (Madison, WI). The mutated proteins are indicated by the one-letter-code for mutation of amino acids. According to a previous study [10], we prepared R19L, Y21V, D22G, R43G, K44S, R45T, and Nd10, in which the N-terminal 10 residues of $\mathrm{P} 12$ were deleted, and $\mathrm{Cd} 8$, in which the $\mathrm{C}$-terminal 8 residues of P12 were deleted. Based on the P12 cDNA, two mutagenic oligonucleotides were also used to create two multisite-directed mutagenesis, namely 5'-CAGGCTCTATGGTACTCCCGTTTTCAAAGC-3' (R43G/ $\mathrm{K} 44 \mathrm{~S} / \mathrm{R} 45 \mathrm{~T})$ and $5^{\prime}$-GAATTCTCAGCAAGGCCCACCTGCTCEAA TGTGG-3' (L50H/R52G/K53A), respectively, where the mismatched bases are underlined. We purified the EcoRI/BamHI fragment of P12

A

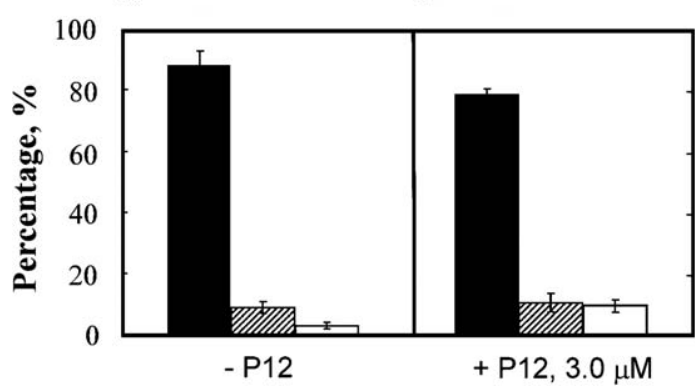

Fig. 1. The impact of P12 on the status of mouse spermatozoa. Freshly prepared mouse sperm from the caudal epididymis in modified Tyrode's solution $\left(2 \times 10^{6}\right.$ cells $\left./ \mathrm{ml}\right)$ devoid of $\mathrm{CaCl}_{2}$ were incubated in the absence of $\mathrm{P} 12$ (A) or in the presence of $\mathrm{P} 12$ (B) in a $5 \% \mathrm{CO}_{2}$ incubator at $37{ }^{\circ} \mathrm{C}$ for $30 \mathrm{~min}$. The CTC fluorescence method described in the text was exploited to score the population of uncapacitated cells (solid bars), capacitated cells (hatched bars), and acrosome-reacted cells (open bars). The effect of P12 on spermatozal status was insignificant. Data represent the means of five individual trials counting 200 cells per trial and error bars represent the SD.
cDNA from an expression vector (Gfp), which had been previously constructed by the insertion of P12 cDNA into pGEX-2T [8] and then inserted the DNA fragment into pALTER-1. Using single-stranded DNA of the recombinant phagemid DNA as a template, the mutant strand containing each mutagenic oligonucleotide was completed according to the manufacturer's recommendations. The phagemid from each selected transformed colony was sequenced to confirm the mutation. The EcoRI/ Bam HI fragment of each P12 variant cDNA was purified and ligated into

A
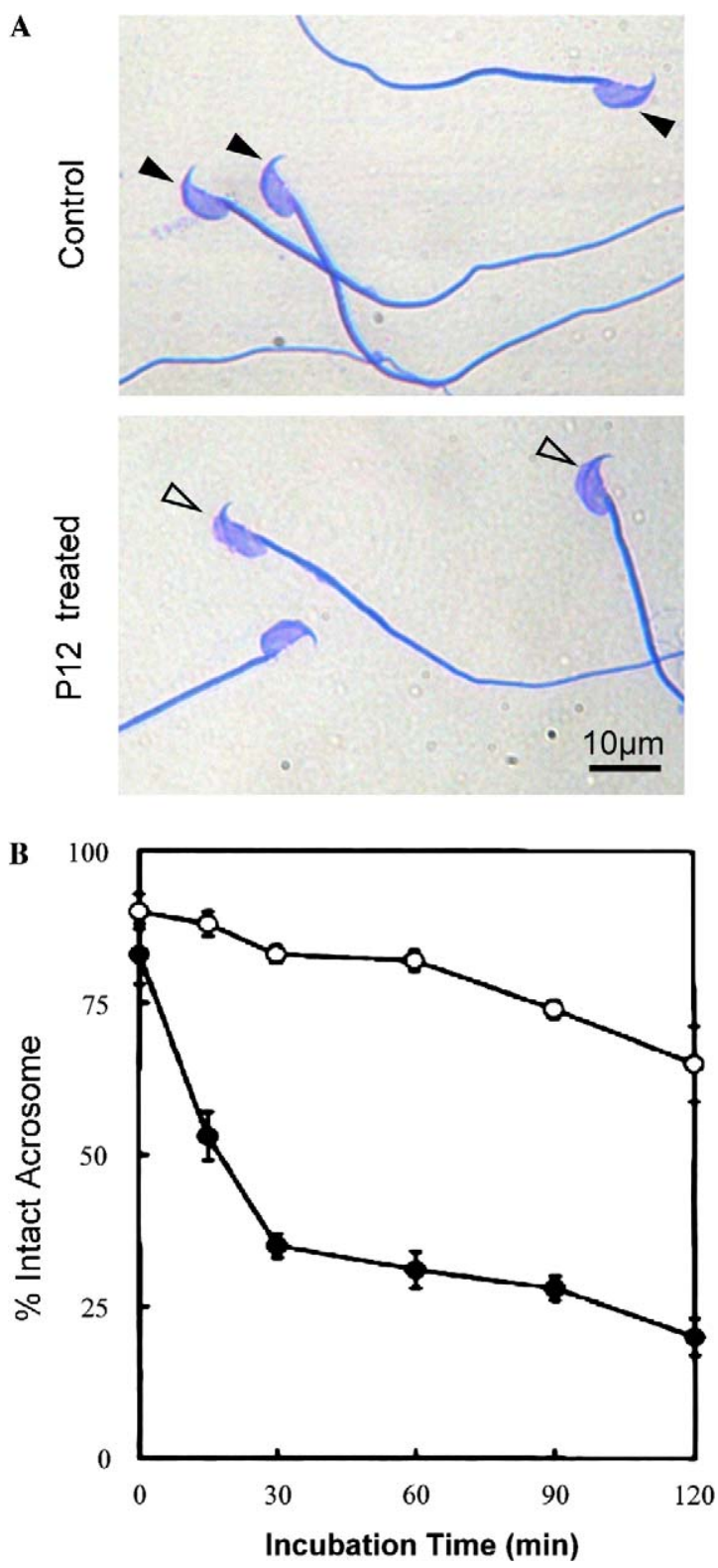

Fig. 2. The change of acrosomal morphology by immunoaggregation of P12 on mouse sperm. Freshly prepared sperm (control, $\bigcirc$ ) and the cells preincubate with $3 \mu \mathrm{M}$ P12 for $10 \mathrm{~min}$ (P12-treated, $\bullet$ ) were exposed to partially purified rabbit P12 antibody $(200 \mathrm{ng} / \mu \mathrm{l})$ in PBS at $\mathrm{pH} 7.2$ for 0 $120 \mathrm{~min}$ at $37^{\circ} \mathrm{C}$. (A) The acrosomal zones of cells were Coomassiestained at $30 \mathrm{~min}$ incubation (details in Materials and methods). The condensed-and-thick-staining zone is indicated by a soli arrow and the thin crescent band in the Coomassie-staining loose zone by an open arrow. (B) The acrosomal status of the sperm was scored according to the Coomassie-staining patterns as described in the text. The data represent the average of three determinations in which 200 cells were counted per determination, and error bars represent the SD. 
pGEX-2T, which was transformed into the E. coli strain JM109. Expression of the GST-fusion protein, purification of the recombinant protein through an affinity column of glutathione agarose beads (Sigma, St. Louis, MO), thrombin digestion of the GST-fusion protein, and purification of the P12 mutant from the thrombin digests via HPLC on a C4 300A column (Waters, Bedford, MA) were performed according to the previously described technique [10].

Examination of the immunoagglutination caused by P12 on mouse sperm. The rodent antisera against P12 were prepared according to our previous study [9]. The antibody was partially purified from the antiserum through an affinity column of protein A. Spermatozoa were extruded from the distal portion of the caudal epididymis and were prepared according to a previously described method [12]. Under the specified conditions, sperm were incubated with $3 \mu \mathrm{M}$ P12 in PBS alone or in the presence of partially purified antibody $(200 \mathrm{ng} / \mu \mathrm{l})$ at $37^{\circ} \mathrm{C}$. Cells were air-dried on a glass slide and washed twice with PBS before cytochemical staining. The slides were immersed in $3.5 \%$ perchloric acid containing $0.04 \%$ Coomassie G 250 for 5 min $[13,14]$. The slides were gently washed with PBS and covered with PBS/glycerol (1:1 by volume). The Coomassie-stained cells were observed under a microscope (AHBS3; Olympus, Tokyo, Japan). Two hundred cells were randomly selected and scored for acrosome integrity.

The chlortetracycline staining method of Ward and Storey [15] was applied to distinguish mouse spermatozoa into uncapacitated, capacitated, or acrosome-reacted stages. The cell morphology was observed under a fluorescence microscope (AHBS3; Olympus). Two hundred cells were randomly selected and scored for each of these three stages.

\section{Results}

\section{Immunoaggregation of P12 on mouse sperm}

The chlorotetracycline-staining assay showed that around $90 \%$ of freshly prepared spermatozoa from caudal epididymis of mice remained uncapacitated, and fewer than $5 \%$ of the cells became acrosome-reacted. When the cells were incubated in modified Tyrode's Solution devoid of
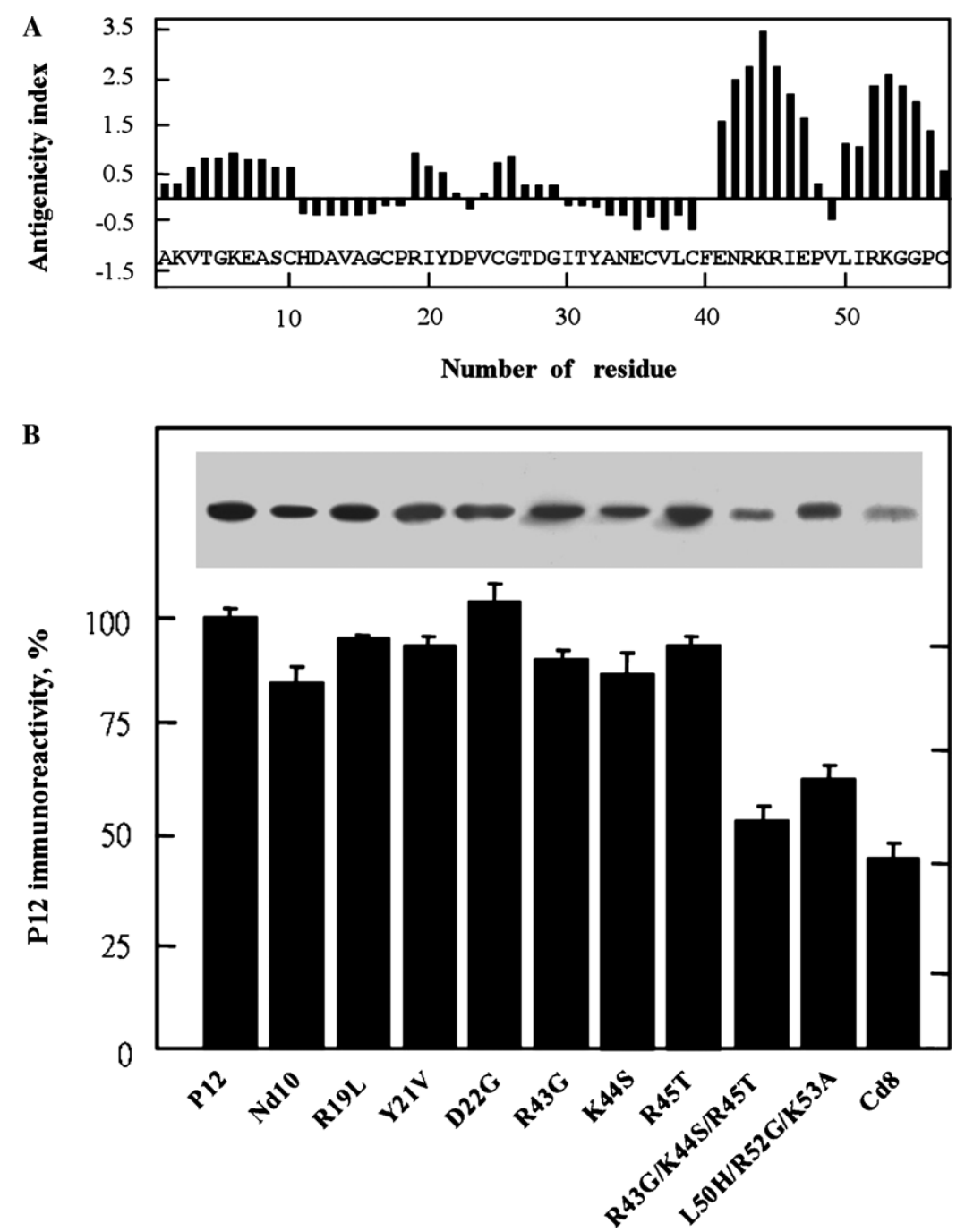

Fig. 3. Three epitopes on the P12 molecule. (A) A prediction of the potential epitopes along the amino acid sequence. (B) Immunoreactivity of the ten P12 variants. Equal amount of each purified protein sample was subjected to SDS/PAGE on a $15 \%$ polyacrylamide gel slab $(6.5 \times 10.5 \times 0.075 \mathrm{~cm})$. The gel was stained with Coomassie brilliant blue (not shown) and immunodetected by Western blot using the P12 antibody from the rabbit antiserum as the primary antibody and horseradish peroxidase-conjugated anti-rabbit IgG as the secondary antibody (top panel). The intensity of each immunoblot band was normalized to its corresponding protein band intensity, and the immunoreactivity of each P12 variant was calculated relative to that of the parent protein, which is referred to as $100 \%$. Error bars represent the SD of the means for three determinations. 
$\mathrm{CaCl}_{2}$ at $37^{\circ} \mathrm{C}$ for $120 \mathrm{~min}$, about $70 \%$ of cells remained uncapacitated. At any incubation time, the cell status was slightly influenced by the presence of $3.0 \mu \mathrm{M} \mathrm{P} 12$ in the cell incubation medium. For instance, more than $80 \%$ of the control cells remained uncapacitated and fewer than $5 \%$ of the cells became acrosome-reacted after 30-min incubation (panel A of Fig. 1). Under the same incubation time, the presence of $3 \mu \mathrm{M}$ P12 in the cell incubation produced a slight effect on the cell status (panel B of Fig. 1). Meanwhile, we examined the Coomassie-staining membrane overlaying on the acrosome region of cells at $30 \mathrm{~min}$ incubation (Fig. 2A). There appeared a condensed-and-thickCoomassie-staining zone on the acrosome of most of the control cells, representing the intact acrosome being retained on the uncapacitated cells. On the other hand, a thin crescent band in the Coomassie-staining loose zone was seen on the acrosome of most of cells being preincubated with P12 for 10 min followed by exposure to the antibody for $30 \mathrm{~min}$, suggesting the removal of plasma membrane overlaying the acrosome by immunoaggregation of P12 on sperm head. The extent of immunoaggregation during the course of cell incubation for $0-120 \mathrm{~min}$ caused a rapid decrease in the population of cells with intact acrosomes over the $0-30 \mathrm{~min}$ cell incubation (Fig. 2B, -). At $30 \mathrm{~min}$ immunoreaction, less than $30 \%$ of the cells retained the intact acrosome. This characteristic was in contrast to the progressive decrease in the cell population with intact acrosome when the cells were incubated in the absence of antibody (Fig. 2B, O); more than $80 \%$ of cells retained intact acrosome at $30 \mathrm{~min}$ incubation.

\section{Identification of the P12 epitopes}

Identification of epitopes on the P12 molecule is indispensable to understand how the P12 antibody is able to immunoreact P12 on the sperm head. Presuming the formation of an epitope on the P12 molecule by more than three consecutive residues with each having an antigenicity index higher than 0.5 , we predicted a weak epitope on residues 3-10 and two strong epitopes on residues $41-47$ and residues 50-57, but none for residues 11-40 according to the method of Jameson-Wolf [16] (Fig. 3A). Based on this prediction, we prepared ten $\mathrm{P} 12$ variants (see Materials and methods). The results of Western analysis shown in the inset of Fig. 2B demonstrated that all the mutants were immunostained by the P12 antibody. Their immunoreactivity to the antibody was compared (Fig. 3B). Nd10, L50H/R52G/R53V, and Cd8 retained 82,62 , and $44 \%$ of the P12 immunoreactivity, respectively. Although R53G, K44S, and R45T each showed 87$94 \%$ of the P12 immunoreactivity, the multisite mutation $\mathrm{R} 43 \mathrm{G} / \mathrm{K} 44 \mathrm{~S} / \mathrm{R} 45 \mathrm{~T}$ caused a great reduction to $52 \%$ of the P12 immunoreactivity. R19L, Y21V, and D22G showed virtually the same strength of immunoreactivity as P12 did. Apparently, the immunoreactive amino acid residues were in accord with those predicted from the antigenicity indices.

\section{Discussion}

This work together with our previous study [10] sheds light on the location of multi-functional sites on P12. According to the molecular model (Fig. 4), the three epitopes are on the side opposite to the location for spermbinding site. The topology of these functional sites enables us to elucidate the structural basis of P12-sperm binding that is suited to immunoaggregation of $\mathrm{P} 12$ on the acrosome of sperm. The binding of D22/Y21 on the P12 molecule to the sperm head from one direction would turn R19 towards the other direction and expose the three epitopes. Such a structural feature allows not only the immunoaggregation of P12 on the sperm head, which causes the removal of membrane overlaying acrosome (Fig. 2), but also the binding of a trypsin-like protease to the reactive R19 from the other direction. Among the sexual organs of male and female, P12 is predominantly secreted from the luminal epithelium of male accessory sexual glands, and the P12sperm binding takes place after ejaculation. Since the ejaculated spermatozoa reside for a significant period in the female reproductive tract of naturally inseminated mice, in particular in the uterine lumen to carry on capacitation, induction of a sufficient amount of P12 antibody in the female reproductive tract is likely to immunoaggregation P12 on the ejaculated sperm before sperm-egg encounter. Thereby, the sperm would become infertile.

Immunocontraception has received increased interest in the past few decades. As our knowledge of molecular reproduction grows, more members of the functional molecules, which participate in the fertilization, development or transportation of gametes, have been assessed as potential candidates for the development of antifertility vaccines.

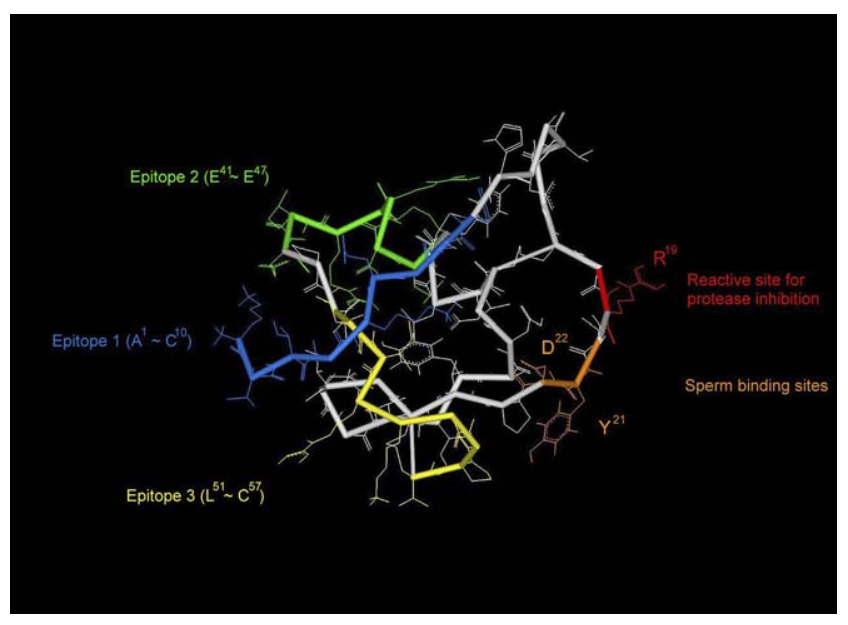

Fig. 4. The topology of functional sites on the P12 molecule. According to the molecular model of P12, the tubing diagram shows the polypeptide backbone consisting of one $\alpha$-helix, which stretches from $\mathrm{E}^{35}$ to $\mathrm{R}^{43}$, and a small antiparallel $\beta$-sheet, which is comprised of the three twisted strands of ${ }^{23} \mathrm{PVCG}^{26},{ }^{27} \mathrm{TDGI}^{30}$, and ${ }^{53} \mathrm{KGGP}^{56}$. The side chains of amino acid residues are displayed using a stick structure. The three epitopes (green, blue, and yellow) are distant from both the sperm-binding site $\left(\mathrm{Y}^{21}\right.$ and $\mathrm{D}^{22}$, orange) and the reactive site for protease inhibition $\left(\mathrm{R}^{19}\right.$, red). 
In this regard, most of the studies thus far have focused on the immunoresponse of reproductive protein hormones $[17,18]$ and gamete-specific antigens $[19,20]$. However, serious side effects, such as hormone imbalance, orchiditis or ovaritis, may develop [21]. Using P12, which is secreted from the male accessory sexual glands, as the contraceptive vaccine may avoid the problems mentioned above. Such a conceptual strategy awaits future study.

\section{References}

[1] H. Tschesche, B. Wittig, G. Decker, W. Muller-Esterl, H. Fritz, A new acrosin inhibitor from boar spermatozoa, Eur. J. Biochem. 126 (1982) 99-104.

[2] M.L. Huhtala, Demonstration of a new acrosin inhibitor in human seminal plasma, Hoppe Seylers Z. Physiol. Chem. 365 (1984) 819-825.

[3] D. Cechova, V. Jonakova, Bull seminal plasma protease inhibitors, in: L. Lorand (Ed.), Academic Press, New York, 1981, pp. 729-803.

[4] S.F. Pang, P.H. Chow, T.M. Wong, The role of the seminal vesicles, coagulating glands and prostate glands on the fertility and fecundity of mice, J. Reprod. Fertil. 56 (1979) 129-132.

[5] B. Peitz, P. Olds-Clarke, Effects of seminal vesicle removal on fertility and uterine sperm motility in the house mouse, Biol. Reprod. 35 (1986) 608-617.

[6] M.L. Lai, S.W. Chen, Y.H. Chen, Purification and characterization of a trypsin inhibitor from mouse seminal vesicle secretion, Arch. Biochem. Biophys. 290 (1991) 265-271.

[7] J.S. Mills, M. Needham, T.C. Thompson, M.G. Parker, Androgenregulated expression of secretory protein synthesis in mouse ventral prostate, Mol. Cell. Endocrinol. 53 (1987) 111-118.

[8] M.L. Lai, S.H. Li, Y.H. Chen, Purification and biochemical characterization of a recombinant mouse seminal vesicle trypsin inhibitor produced in Escherichia coli, Protein Expr. Purif. 5 (1994) 22-26.

[9] L.Y. Chen, Y.H. Lin, M.L. Lai, Y.H. Chen, Developmental profile of a caltrin-like protease inhibitor, P12, in mouse seminal vesicle and characterization of its binding sites on sperm surface, Biol. Reprod. 59 (1998) 1498-1505.

[10] C.W. Luo, H.J. Lin, S.C. Gopinath, Y.H. Chen, Distinction of sperm-binding site and reactive site for trypsin inhibition on 12 secreted from the accessory sex glands of male mice, Biol. Reprod. 70 (2004) 965-971.

[11] C.W. Luo, H.J. Lin, Y.H. Chen, A novel heat-labile phospholipidbinding protein, SVS VII, in mouse seminal vesicle as a sperm motility enhancer, J. Biol. Chem. 276 (2001) 6913-6921.

[12] Y.H. Huang, S.T. Chu, Y.H. Chen, A seminal vesicle autoantigen of mouse is able to suppress sperm capacitation-related events stimulated by serum albumin, Biol. Reprod. 63 (2000) 1562-1566.

[13] D. Aarons, H. Boettger-Tong, G. Holt, G.R. Poirier, Acrosome reaction induced by immunoaggregation of a proteinase inhibitor bound to the murine sperm head, Mol. Reprod. Dev. 30 (1991) 258-264.

[14] C.C. Moller, J.D. Bleil, R.A. Kinloch, P.M. Wassarman, Structural and functional relationships between mouse and hamster zona pellucida glycoproteins, Dev. Biol. 137 (1990) 276-286.

[15] C.R. Ward, B.T. Storey, Determination of the time course of capacitation in mouse spermatozoa using a chlortetracycline fluorescence assay, Dev. Biol. 104 (1984) 287-296.

[16] B.A. Jameson, H. Wolf, The antigenic index: a novel algorithm for predicting antigenic determinants, Comput. Appl. Biosci. 4 (1988) 181-186.

[17] L.A. Miller, B.E. Johns, D.J. Elias, K.A. Crane, Comparative efficacy of two immunocontraceptive vaccines, Vaccine 15 (1997) 1858-1862.

[18] P.J. Delves, How far from a hormone-based contraceptive vaccine? J. Reprod. Immunol. 62 (2004) 69-78.

[19] A. Domagala, M. Kurpisz, Identification of sperm immunoreactive antigens for immunocontraceptive purposes: a review, Reprod. Biol. Endocrinol. 2 (2004) 11.

[20] S.K. Gupta, S. Chakravarty, S. Kadunganattil, Immunocontraceptive approaches in females, Chem. Immunol. Allergy 88 (2005) 98-108.

[21] S.K. Gupta, N. Srivastava, S. Choudhury, A. Rath, N. Sivapurapu, G.K. Gahlay, D. Batra, Update on zona pellucida glycoproteins based contraceptive vaccine, J. Reprod. Immunol. 62 (2004) 79-89. 